\title{
THE CONTROL OF CROSSING-OVER IN THE $X$-CHROMOSOME OF DROSOPHILA MELANOGASTER
}

\author{
M. J. LAWRENCE \\ Department of Genetics, The University, Birmingham 15
}

\section{INTRODUCTION}

Received 1 3.vi.62

IT has long been established that crossing over varies in the same way as many other features of the phenotype, though the consequence of such variations are, of course, different from those of variation of morphological characters. As long ago as 19r9, Gowen showed that crossing-over in the third chromosome of Drosophila melanogaster was under polygenic control, a conclusion extended more recently by Levine and Levine (1955) to Drosophila pseudoobscura and by Rees to rye (1955). Yet with few exceptions, notably that of Rees, few attempts have been made to design experiments on crossing-over which permit the partitioning of total variation in the way now customary with quantitative characters.

There are, of course, two ways in which crossing-over may be studied, (i) by the cytological observation of chiasma frequency and (ii) by the genetical determination of the frequency of recombination, between linked marker genes of a single chromosome. While the cytological approach provides information concerning the distribution of chiasmata among all the chromosomes of a nucleus, it can rarely tell us much about the distribution of points of exchange along the length of individual chromosomes. Since there is evidence that genes controlling a character are not distributed evenly along the length of a chromosome (Wigan, 1949; Breese and Mather. 1957), the distribution of points of exchange is clearly as important to the evolutinnary future of a species as is the total amount of recombination in the nucleus. Again, although not a limitation inherent in the approach, cytological experiments have been rarely carried out in genetically homogeneous material or, indeed, that convenient for breeding purposes.

With these considerations in mind, two experiments of the same general type have been carried out using several inbred lines of Drosophila melanogaster. In these experiments common, marked Xchromosomes have been put into each inbred line by the technique customary in Drosophila. These inbred lines were then crossed together so that various substitutions of the autosomal genotype could be assessed with respect to their effects on the frequency of recombination between the marker genes of the $\mathrm{X}$-chromosome. It is important to draw a distinction between the X-chromosome and the autosomes here. All progenies which were scored for the purpose of estimating the frequency of recombination between markers on the $\mathrm{X}$-chromosome arose, of 
course, from females heterozygous for their X-chromosomes. These same females, however, were not necessarily heterozygous with respect to their autosomes, this being a controlled variable of the experiment. The terminology "parents " and "FI's" thus refer to the autosomes and not to the X-chromosomes.

The details and results from the first experiment have been reported elsewhere (Lawrence, 1958). Although the purpose of this account is to present the results from the second experiment it is worthwhile mentioning those from the first experiment, since one was the direct outcome of the other. The first experiment was concerned with two inbred lines Oregon $(\mathrm{O})$ and Samarkand $(\mathrm{S})$ and their reciprocal $\mathrm{F}_{\mathrm{I}}$ crosses. The experiment was carried out in each of four environments, $15^{\circ}, 20^{\circ}, 25^{\circ}$ and $28^{\circ} \mathrm{C}$. The X-chromosome used carried three markers, white-eye $(w)$, miniature-wing $(m)$ and Bar eye $(B)$. The design of the experiment was that of a balanced three-point linkage test ( $c f$. Wallace, 1957). No serious or consistent disturbance of the type caused by differential visibility was encountered for any of the three markers and the estimation of linkage was undertaken by the product method.

The three main points of interest emerged from the results of this experiment:-

(I) There were highly significant differences between Oregon and Samarkand and between these and their FI's;

(2) There was also a highly significant difference between the reciprocal $F_{I}$ 's, this effect being largely confined to the distal region of the chromosome $(w-m)$. This difference was in the direction expected if maternal factors were active in determining the frequency of recombination.

(3) The effect of environmental variation was not confined to those regions proximal to the centromere. Regional sensitivity to change of environment varied markedly with genotype. (There was also some evidence of maternal control of regional sensitivity.)

In view of these results, particularly those concerning the environment and maternal determination, it appeared desirable to find out to what extent these were regular features of the control of crossing-over.

\section{MATERIALS AND METHODS}

In the second experiment the X-chromosomes used carried eight marked loci and the genetic analysis was extended to five inbred lines although the experiment was carried out in two environments only $\left(18^{\circ}\right.$ and $25^{\circ} \mathrm{C}$.).

The eight markers used were distributed between two $\mathrm{X}$-chromosomes in such a way that in females heterozygous for these chromosomes adjacent loci were in the repulsion phase (fig. I). This was done both to avoid the difficulty of maintaining inbred lines carrying eight mutant genes and because it was considered impracticable to attempt a balanced multi-point design due to the size of the experiment. Both of these X-chromosomes were put into each of five inbred lines, Oregon $(\mathrm{O})$, Samarkand 
(S), Florida (F), Great Braxted (G) and Edinburgh (E), making five pairs of stocks in all. Thus the $\mathrm{X}$-chromosomes in all lines were standard while the autosomes were line chromosomes.

The genetic analysis of the five lines were undertaken by the diallel cross method (Jinks, 1954) making twenty-five "selfs" and crosses in all. Each self and each cross was raised in duplicate by recognising that the multiply heterozygous females whose meiotic products have been scored, could be made up in two ways viz. $s c c v \vee f \times e c$ ct $s$ car $(\mathrm{I} \times 2)$ and correspondingly ec ct $s$ car $\times s c$ cv $v f(2 \times 1)$. The total number of selfs and crosses possible here is thus fifty. Unfortunately one cross at

\section{Experiment 1}

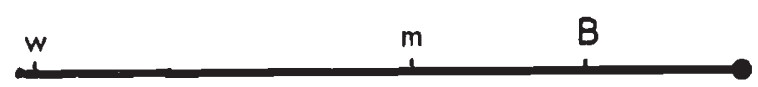

\section{Experiment 2}
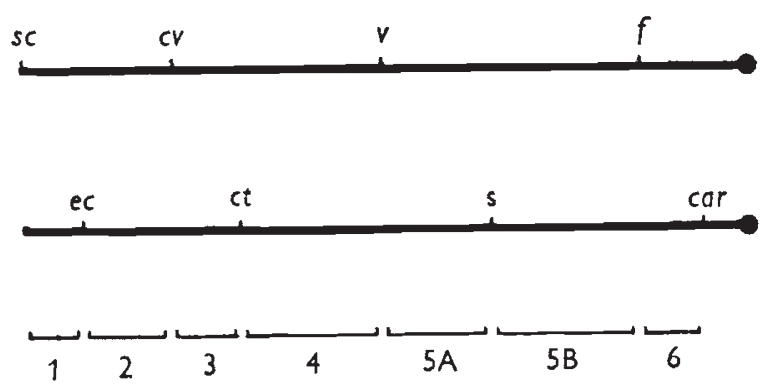

Region

Fro. I.-The marked X-chromosomes used in the two experiments. $w=$ white; $m=$ miniature ; $B=$ bar ; $s c=$ scute ; $c v=$ cross-veinless ; $v=$ vermilion; $f=$ forked ; $e c=$ echinus ; $c t=$ cut ; $s=$ sable ; and $c a r=$ carnation. The chromosomes are drawn to scale, the map distances being those from Bridges and Brehme (1944).

$25^{\circ} \mathrm{C}$., $\mathrm{F} \times \mathrm{G}(\mathrm{I} \times 2)$ and two at $18^{\circ} \mathrm{C}$., $\mathrm{S} \times \mathrm{G}(\mathrm{I} \times 2)$ and $\mathrm{E} \times \mathrm{G}(\mathrm{I} \times 2)$ were not obtained. The entries in the tables corresponding to these missing cultures were filled in from the values of their $2 \times 1$ replicates and the appropriate adjustment made to the degrees of freedom in the analysis of variance.

All multiply heterozygous females were raised in $3 \times \mathrm{I}$ inch tubes. On emergence two days were needed to collect all the females required at $25^{\circ} \mathrm{C}$. and four days at $18^{\circ} \mathrm{C}$. These females were then backcrossed to males of the constitution ... sc ec cv ct of car (hence sable was not scored among the female progeny). Since sable is not accurately classifiable at $25^{\circ} \mathrm{C}$., all backcross progenies were raised at $18^{\circ} \mathrm{C}$. Thus for the experiment carried out at $25^{\circ} \mathrm{C}$., the multiply heterozygous females were raised at $25^{\circ} \mathrm{C}$., held for four days at this temperature as adults (two for the purpose of collection and two for mating) and then transferred to $18^{\circ} \mathrm{C}$. The females were allowed to lay for eight days before being discarded. Thus all the progenies in the experiment at $25^{\circ} \mathrm{C}$. can reasonably be expected to be the products of female meioses at $25^{\circ} \mathrm{C}$. The experiment at $18^{\circ} \mathrm{C}$. was run at this temperature throughout.

Since it was intended that the analysis of the results should be carried out by the analysis of variance, an attempt was made to score 150 flies of each sex from each 
culture. Not all cultures yielded this number, the average number scored being J 40 flies of each sex per culture.

Finally, the salivary gland chromosomes of all five parents and the ten Fr's between them were examined beforehand for the purpose of guarding against the possibility that their chromosomes might be heterozygous for major inversions. In fact, no evidence of any chromosomal rearrangement was gained by this precaution.

\section{DIFFERENTIAL VIABILITY}

The percentage viability of each marker gene (gained as the proportion of mutant phenotype to total number of individuals scored in a culture) was obtained for each sex and each culture raised in the

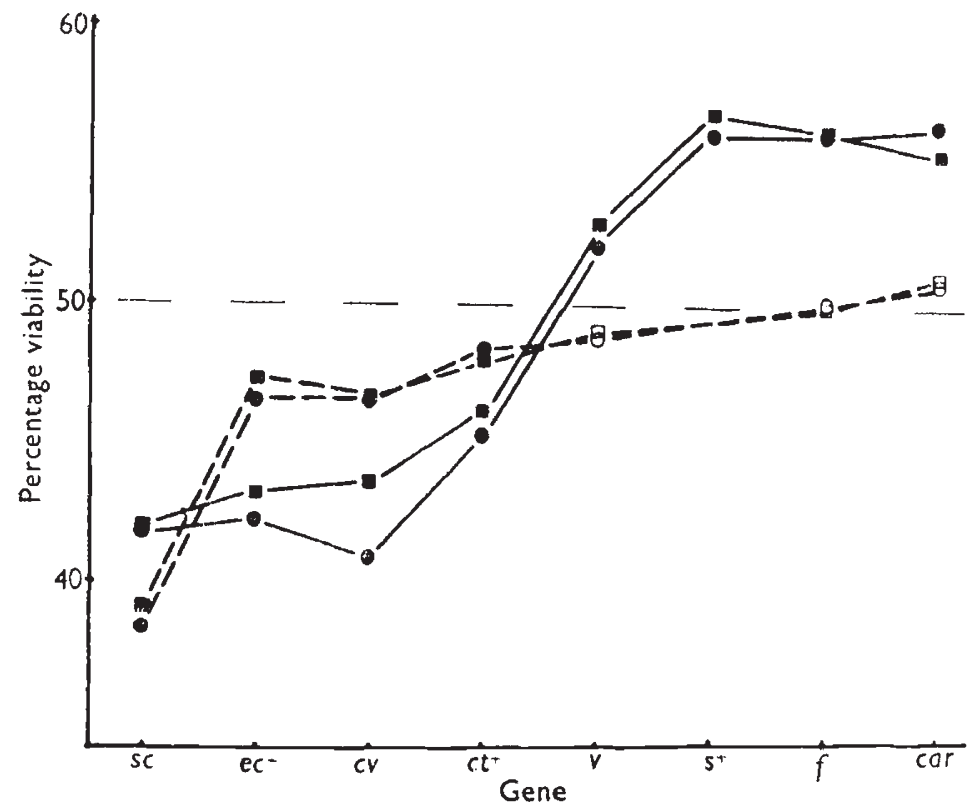

FIG. 2.- The percentage viabilities of the marker genes used in the second experiment.

Full lines $=$ males; broken lines $=$ females ; squares $=18^{\circ} \mathrm{C}$. experiment ; circles $=25^{\circ} \mathrm{C}$. experiment. Blocked-in squares and circles indicate significant departures from the I : I ratio.

experiment. The mean percentage viabilities obtained by averaging over cultures are shown with respect to the sc cv $v f$ chromosome in fig. 2. It is clear that with the exception of $v, f$ and car in females, all markers show significant departures from the expected I : I ratio.

As would be expected, (since all scoring progenies were raised at $18^{\circ} \mathrm{C}$.) the maternal environment has little effect on viability of the marker genes in either sex. But the viability disturbances among the males of the scoring progeny are obviously different from those among the female progeny. The most reasonable interpretation of the viability curve for males is that at or near $c v$ and $s$ respectively, there are genes of low viability. On this view the remaining markers show viability disturbances because they are linked to these two. Since $c v$ and $s$ are in repulsion, we expect their complimentary recombinant classes in 
different proportions from equality, one recombinant class carrying both the genes, the other carrying neither. A disturbance of the kind predicted was in fact observed among the males of the scoring progenies, the latter recombinant class often being three times as numerous as that carrying both the defective markers. The curves for females on the other hand are expected to differ from those of the males, unless the disturbance at $s$ is dominant, since this marker was not scored among female progeny. Their curves suggest a disturbance at the $c v$ locus only. The apparent low viability of sc is mainly due to partial manifestation of this marker in females and this will be discussed in the next section.

With extensive viability disturbances of this kind and without resort to a balanced design, any estimate of linkage may be biased. In the absence of extreme viability disturbances however-and few of the viabilities in this experiment fell outside the range 40-6o per cent.- the bias will be small. Furthermore, our main interest here is not concerned with the precise determination of mean recombination frequency but rather with variation about the observed mean.

Now since the viability curves differ between sexes, especially in proximal regions of the chromosome, the bias due to viability disturbances will affect the linkage values from male and female progeny differently. If, therefore, in spite of this, their linkage estimates are homogeneous, the bias must be trivial compared with other sources of variation and we may proceed with confidence. In fact, as will be shown later, the sexes are quite homogeneous with regard to their linkage estimates.

\section{PARTIAL MANIFESTATION}

It is well known that disturbances of segregation ratios due to incomplete penetrance or partial manifestation cause a more serious bias of linkage estimates than do those due to differential viability, unless this is very severe. It quickly became apparent that sc was failing to manifest itself among the females of the progenies scored (the males showing no disturbance of this kind). Two small independent manifestation tests were carried out to check this view. These showed that about 16 per cent. of females known to be homozygous for the scute gene failed to manifest the scute phenotype. The degree of manifestation moreover varied significantly between genotypes thus confirming the analysis of the scute segregation ratio in the main experiment.

Now, while methods of estimating linkage in the presence of partial manifestation have been developed (Parsons, 1957; Allard and Alder, I 960), the problems of estimation when both partial manifestation and differential viability are present have received less attention.

A further problem, given that efficient estimates of linkage could be obtained under these circumstances, concerns the appropriate transformation to be used when we wish to proceed to the analysis of 
variance. It seems unlikely that the variances of the transformed estimates would any longer be simple function of the number scored as they are, of course, when the angular transformation is used for this purpose. The estimation of linkage has not therefore been carried out for the $s c-e c$ region of the X-chromosome among the female progenies, such information as we have here springing solely from the male progenies.

\section{RESULTS}

The estimation of linkage was undertaken by the simple percentage comparison of number of recombinant types to total number scored. Estimates were then transformed into angles so that the analysis of the results could be carried out by means of the analysis of variance of diallel tables (Hayman, 1954).

Since the experiment, a full $5 \times 5$ diallel, was duplicated and carried out in each of two environments, one hundred cultures would have been raised in all. Recalling that three cultures failed, however, the total was reduced to ninety-seven and hence a total of 96 degrees of freedom are available for comparisons between cultures. Since males and females were classified in each culture scored, 97 degrees of freedom are available for within culture, between sex, comparisons.

Linkage estimates were obtained from seven chromosome regions from male and from five regions in female progenies. Comparisons between sexes are thus possible for only five regions-those common to both sexes.

The main items in Hayman's analysis are, briefly:-

(I) Additive genetic variation or general combining ability, $a$;

(2) Non-additive genetic variation or special combining ability, $b$;

(3) Average maternal effects of each line, $c$;

(4) Reciprocal differences not ascribable to $c, d$.

The dominance item, $b$, may be further partitioned into three items:-

(I) Average dominance, $b_{1}$;

(2) Average dominance differences among parental lines, $b_{2}$;

(3) Residual dominance effects, $b_{3}$.

In general, the $b_{2}$ and $b_{3}$ items have not been shown in the tables as they were not significant.

The results from each segment, each sex, each duplicate and each environment were analysed by this method, heterogeneity between sexes, duplicates and environments being investigated by pooling the data in the usual way. The results of this analysis are shown in tables IA and І в. We may also pool over segments so as to gain a table whose entries reflect the total amount of recombination occurring in the chromosome. However, it is not possible to investigate interactions between segments and genotypes in this way since recombinational 
CONTROL OF CROSSING-OVER

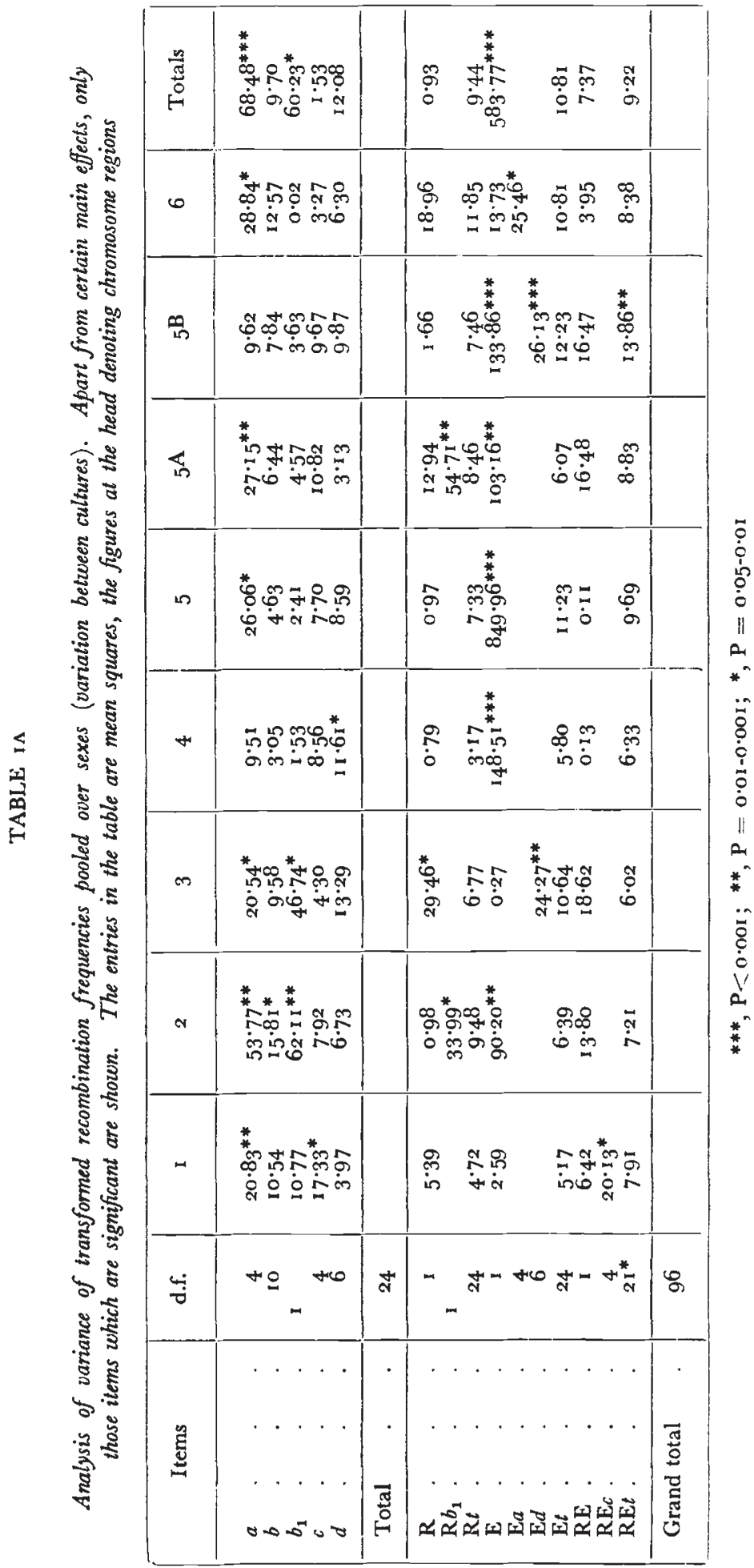

C 
events are clearly not independent in adjacent segments. The analysis of totals appears in the last column of tables IA and IB.

Before proceeding to discuss these analyses, it is necessary to explain the source of the error mean squares used in the tests of significance. Provided that any summation process is restricted to replicates or environments (or both) we have an expected error mean square due to the angular transformation used. The analysis of unsummed data and that where summation is carried out over replicates or environments only thus leads to the chi-squared test of significance. Where,

TABLE IB

Analysis of variance of transformed recombination frequencies (variation within cultures). See previous table for details

\begin{tabular}{|c|c|c|c|c|c|c|c|}
\hline Items & d.f. & 2 & 3 & 4 & 5 & 6 & Totals \\
\hline Sex & I & $4 \cdot 76$ & 0.01 & 4.01 & $17 \cdot 70$ & $59 \cdot 30^{* *}$ & $5^{\mathrm{I}} \cdot 03^{* *}$ \\
\hline $\begin{array}{l}\mathrm{S} a \\
\mathrm{~S} b \\
\mathrm{~S} b_{1} \\
\mathrm{~S} c \\
\mathrm{~S} d\end{array}$ & $\begin{array}{r}4 \\
10 \\
1 \quad \\
4 \\
6\end{array}$ & $\begin{array}{c}6 \cdot 05 \\
9 \cdot 64 \\
51.55^{*} \\
6.25 \\
6.03\end{array}$ & $\begin{array}{l}8 \cdot 68 \\
3 \cdot 5^{6} \\
2 \cdot 10 \\
3 \cdot 4^{6} \\
4 \cdot 55\end{array}$ & $\begin{array}{c}11 \cdot 52 \\
7 \cdot 40 \\
2 \cdot 30 \\
10 \cdot 46 \\
16 \cdot 68 *\end{array}$ & $\begin{array}{r}10 \cdot 12 \\
6 \cdot 16 \\
4 \cdot 88 \\
9 \cdot 14 \\
1 \cdot 79\end{array}$ & $\begin{array}{l}4 \cdot 28 \\
6 \cdot 72 \\
1 \cdot 86 \\
5 \cdot 73 \\
9 \cdot 91\end{array}$ & $\begin{array}{r}10.32 \\
5.68 \\
8 \cdot 16 \\
8.98 \\
3.85\end{array}$ \\
\hline St & 24 & & & & & & \\
\hline $\mathrm{SR}$ & I & I.05 & II $\cdot 98$ & $4 \cdot 20$ & 19.53 & $I \cdot 25$ & $5 \cdot 31$ \\
\hline $\begin{array}{l}\mathrm{SR} b_{1} \\
\mathrm{SR} t \\
\mathrm{SE} \\
\mathrm{SE} t \\
\mathrm{SRE} \\
\mathrm{SRE} t\end{array}$ & $\begin{array}{rr}I & \\
& 24 \\
& \\
& 24 \\
& I \\
& 21\end{array}$ & $\begin{array}{l}8 \cdot 72 \\
0 \cdot 11 \\
9 \cdot 36 \\
8 \cdot 04 \\
7 \cdot 83\end{array}$ & $\begin{array}{l}4 \cdot 62 \\
8 \cdot 19 \\
6 \cdot 86 \\
1 \cdot 33 \\
7 \cdot 03\end{array}$ & $\begin{array}{r}6.00 \\
10.5^{8} \\
5.42 \\
0.03 \\
6.4^{2}\end{array}$ & $\begin{array}{r}75 \cdot 34^{*} \\
7.73 \\
3.89 \\
7.04 \\
18.06 \\
12.20\end{array}$ & $\begin{array}{l}9 \cdot 89 \\
1 \cdot 18 \\
3 \cdot 43 \\
6 \cdot 31 \\
8 \cdot 39\end{array}$ & $\begin{array}{l}6 \cdot 97 \\
0 \cdot 01 \\
6 \cdot 93 \\
5.51 \\
5 \cdot 30\end{array}$ \\
\hline $\begin{array}{c}\text { Grand } \\
\text { total }\end{array}$ & 97 & & & & & & \\
\hline
\end{tabular}

however, the analysis concerns data obtained by summing over sexes and/or segments, the appropriate error mean square for comparisons between cultures is REt and for those within cultures is SRE $t$, since neither sex or segment are independent. As is usual, whenever lower order interactions in the analysis turn out to be neither significant nor heterogeneous the error mean squares used to test main effects have been obtained by pooling these with those items just mentioned.

Turning now to the analysis of variation between sexes (table IB) only one item is of such significance that it appears in the analysis of totals; that concerned with the average difference between sexes in region 6 . This is perhaps hardly surprising since it will be recalled that in this region the difference between male and female viabilities was maximal and it is difficult to account for heterogeneity between sexes with respect to their linkage estimates in any other way here.

Three further items within the body of table I B are also significant. Before attempting to interpret these, however, it is worth while enquiring 
how many such items are to be expected on the basis of sampling variation. Now in the full table there are $100(5 \times 20)$ orthogonal mean squares. Due to sampling variation alone we expect on average 5 of these to be significant at the 5 per cent. level and I at the I per cent. level. In fact, we find 3 and I respectively, so that there appears to be little doubt that the sexes are homogeneous with respect to their linkage estimates. Thus despite the known differences between sexes with regard to their differential viabilities, such bias as this might introduce in the estimation of linkage must be relatively trivial.

It is clear from the last column of table IA that only three items are of importance with respect to the total amount of crossing over within the $\mathrm{X}$-chromosome here; additive genetic variation $(a)$, mean dominance $\left(b_{1}\right)$ and the environment $(E)$. A partitioning of the additive item reveals that the major comparison in this respect lies between Florida, with a high overall frequency of recombination, and Edinburgh with a low overall frequency. The other lines are essentially intermediate to these two. The significance of the dominance item is due to the higher recombination frequency of the FI's when these are compared with their inbred parents, while that of the environment is due to the generally higher frequency of recombination at $18^{\circ} \mathrm{C}$. compared with that at $25^{\circ} \mathrm{C}$. Lastly, although not quite significant, the $\mathrm{E} a$ item is large indicating, perhaps, the presence of genotypeenvironment interaction.

Now although the results with respect to the total amount of crossing over on the X-chromosome are of some interest, the manner in which this varies from one segment to another is more relevant to the purpose of this experiment. Since, as already mentioned, we are unable to estimate such variation as interactions between main effects and segments, we shall approach this problem by simply comparing the results of analyses carried out on the individual segments (a multiple regression analysis of recombination frequency on segment could be useful here but it was decided that the amount of information that might be gained in this way scarcely outweighed the very considerable labour involved).

Returning to table IA, it is clear that, with the exception of regions 4 and $5^{B}$, additive genetic variation is present in respect of the entire length of the $\mathrm{X}$-chromosome. As the analysis of totals indicated, the main comparison here lies between Florida and Edinburgh arrays. In region 3, however, the major contrast lies between two groups of parents, one containing Florida and Edinburgh, the other containing the remaining arrays (fig. 3 ). Just why regions 4 and $5^{B}$ appear to be anomalous in that they do not show any significant effect of parental genotype is not clear, although two reasons may be proposed. Regions $5^{\mathrm{B}}$ is delimited by $s$ and $f$, the former being one of the two marker genes showing a disturbed segregation ratio. It is conceivable that in some way the differential viability of $s$ is obscuring the differences between lines with respect to their recombination frequencies. But region 4 
is some way removed from either of the "bad" genes so that this explanation can scarcely be made to serve both regions 4 and $5^{\mathrm{B}}$. On the other hand these regions are the longest of the total of seven examined, both being about thirteen standard map units in length. Thus the probability of the occurrence of undetected double crossing over is higher in these regions (although still very low) than elsewhere. It is therefore possible that this cause has led to a loss in discrimination between lines.

Dominance with respect to crossing over on the X-chromosome appears to be confined to distal regions, i.e. regions 2 and 3 (see fig. 5).

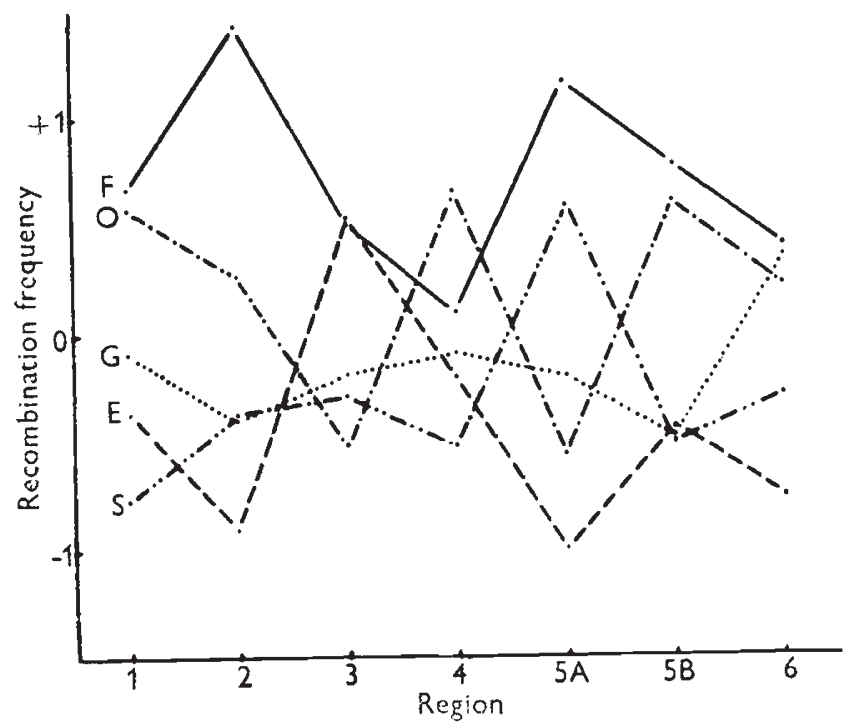

Fic. 3.-The relative frequency of recombination for each of the seven regions of the $\mathrm{X}$-chromosome examined and for each of the five parents used. The ordinates are array deviations from the mean frequency of recombination for that segment, the arrays being averaged over both environments. $E=$ Edinburgh ; $F=$ Florida ; $G=$ Great Braxted ; $\mathrm{O}=$ Oregon; and $\mathrm{S}=$ Samarkand. (Regions $1,5 \mathrm{~A}$ and $5 \mathrm{~B}$ from male progenies only).

The evidence from region 2 should, however, be treated with some caution for dominance is neither consistent over replicates $\left(R b_{1}\right.$ being significant) nor, if the significance of $S b_{1}$ is taken at its face value, over sexes. In any case the role of dominance is clearly subsidiary to that of additive variation in this context.

Environmental variation has a marked effect on the frequency of recombination over the major part of the chromosome (fig. 4). A most surprising feature of the results here is the absence of any detectable effect in the most proximal segment, 6 . Yet perhaps the most consistent feature of previous studies concerned with the effect of the environment on the frequency of recombination, is that those regions proximal to the centromere are the most sensitive to changes of environment. The present results evidently require some explanation, a matter to which we shall return in a later section. 


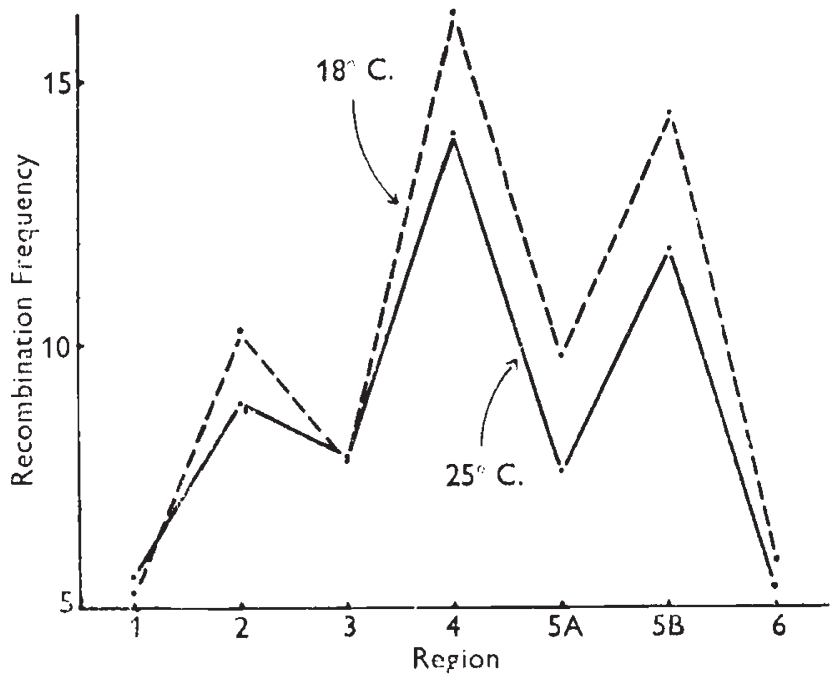

Fig. 4.-The frequency of recombination for each segment in each environment. (Regions I, $5 \mathrm{~A}$ and ${ }_{5} \mathrm{~B}$ from male progenies only.)

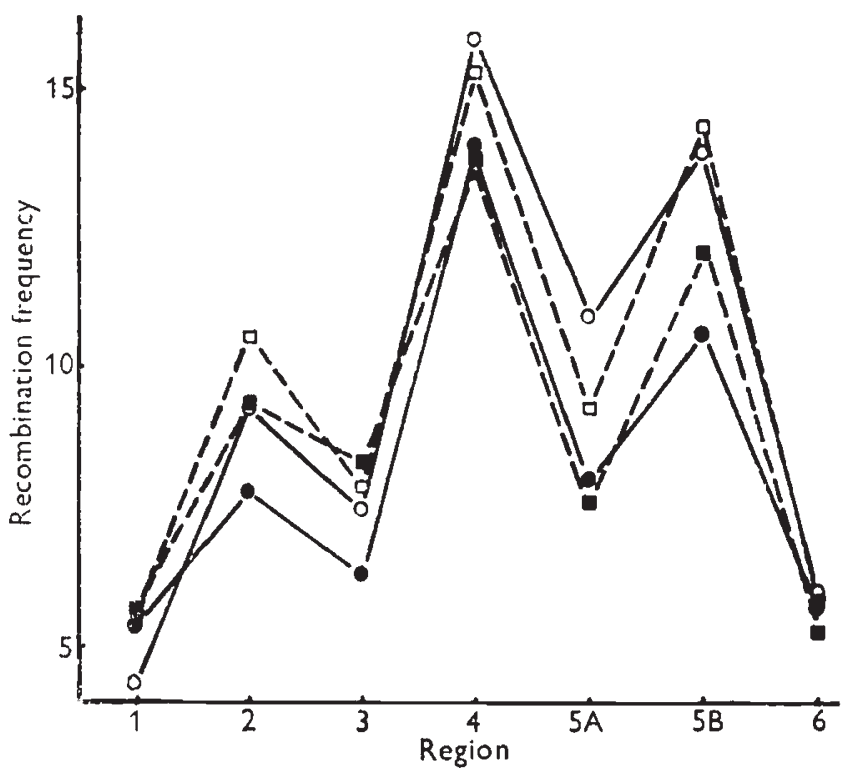

Fig. 5.-The frequency of recombination for each segment in each environment among inbred parents and their hybrids. Full lines and circles = parents ; broken lines and circles $=F_{1}$ 's. Open squares and circles $=18^{\circ} \mathrm{C}$.; blocked-in squares and circles $=25^{\circ} \mathrm{C}$.

The failure of segment 3 to respond, although less surprising than that of segment 6 , is equally inexplicable, while that of segment I could be reasonably taken to indicate a tailing-off effect at the end of the chromosome. These exceptions apart, there is no doubt that the environment must be considered to be a major component of variation over most of the length of this chromosome and not just that part near the centromere. 
Despite the peculiarity of segment 6 in the respect just noted it is this segment alone which provides evidence of genotype-environment interaction. The frequency of recombination in Oregon and Florida arrays appear to be negatively correlated with temperature, while that of the remaining arrays shows almost no correlation.

A major feature of the results of the first experiment was the marked effect of maternal inheritance in distal regions of the chromosome. The present experiment allows a more detailed analysis of reciprocal difference of which maternal effects are a special case. The general test for reciprocal differences here is obtained by pooling the $c$ and $d$ mean squares and this is comparable to the test for maternal effects in the first experiment. In two regions ( $\mathrm{I}$ and 4 ) this comparison is significant-but not elsewhere. In region I the significance of the pooled mean square is due to the $c$ item alone. This implies some uniformity in direction and magnitude of the reciprocal difference among members of the same array but does not tell us the direction of these differences i.e. whether they are the result of matroclinous or patroclinous inheritance. This can be ascertained from the data from which the $c$ mean square was obtained. In terms of direction, six of the ten reciprocal pairs of $\mathrm{F}_{\mathrm{I}}$ 's show a matroclinous difference. But the average magnitude of these is only a little greater than those showing a patroclinous difference.

In region 4 it is the $d$ item which is responsible for that of the pooled mean square. In this case the members of the same array do not in general show reciprocal differences similar either in direction or magnitude. Again, however, we must examine the data to decide whether these differences are on average matroclinous or patroclinous. Six of the ten $F_{I}$ pairs do in fact display a matroclinous difference, as in region I, but only three of these are common to both regions. In terms of magnitude, however, the differences between those showing patrocliny are half as large again as those showing matrocliny. Overall and in detail then it is clear that evidence of maternal inheritance is more conspicuous by its absence in this experiment. It is, however, equally clear that some crosses do display reciprocal differences which, both in terms of magnitude and direction would, if taken on their own, indicate persuasive evidence of maternal inheritance. It is thus reasonable to suppose that this was the situation found in the first experiment. Unfortunately, in the present experiment we have no consistent supporting evidence of matroclinous inheritance in crosses between Oregon and Samarkand. Two distal segments do in fact display a matroclinous difference, but two others exhibit patrocliny in this respect. The results gained from the first experiment with regard to maternal inheritance are not therefore substantiated by those here.

A suggestion of the environmental modification of reciprocal differences is provided by regions 3 and ${ }_{5} \mathrm{~B}$, in both of which the $\mathrm{E} d$ item is significant. In the absence of more compelling evidence from other regions, however, this too should be treated with caution. 
Although it is clear from the analyses presented so far that the main effects are consistent over environments, it is worth pressing the enquiry further by considering the results of the analyses carried out on the data prior to pooling over environments. These are shown in tables 2 and 3 . With the exception of the two most distal segments, there is

\section{TABLE 2}

The analyses of the results from the experiment carried out at $18 \mathrm{C} .^{\circ}$

Other details, as in previous tables

(The interaction of the main effects with sex are homogeneous and are therefore not shown)

\begin{tabular}{|c|c|c|c|c|c|c|c|c|c|c|c|}
\hline Items & & d.f. & 1 & 2 & 3 & 4 & 5 & $5^{\mathrm{A}}$ & $5^{B}$ & 6 & Totals \\
\hline $\begin{array}{ll}a & . \\
b \\
b_{1} & . \\
c & . \\
d & .\end{array}$ & 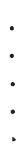 & $\begin{array}{r}4 \\
10 \\
1 \quad \\
4 \\
6 \\
6\end{array}$ & $\begin{array}{r}5 \cdot 18 \\
6 \cdot 32 \\
17 \cdot 57 \\
9 \cdot 92 \\
7 \cdot 04\end{array}$ & $\begin{array}{c}29 \cdot 88^{* *} \\
8 \cdot 96 \\
23.17 \\
6.83 \\
6.06\end{array}$ & $\begin{array}{c}15 \cdot 18^{*} \\
4 \cdot 07 \\
1 \cdot 70 \\
8 \cdot 3^{8} \\
31 \cdot 09^{* * *}\end{array}$ & $\begin{array}{l}8 \cdot 51 \\
1 \cdot 00 \\
3 \cdot 00 \\
8 \cdot 67 \\
9 \cdot 77^{*}\end{array}$ & $\begin{array}{r}27 \cdot 87^{*} \\
5.53 \\
1 \cdot 90 \\
17 \cdot 14 \\
7.66\end{array}$ & $\begin{array}{c}34 \cdot 64^{* *} \\
10 \cdot 82^{*} \\
6 \cdot 34 \\
3.51 \\
3 \cdot 69\end{array}$ & $\begin{array}{c}13 \cdot 24 \\
4 \cdot 79 \\
1 \cdot 32 \\
2 \cdot 02 \\
13 \cdot 26^{*}\end{array}$ & $\begin{array}{c}42 \cdot 98^{* *} \\
13 \cdot 97 \\
2 \cdot 74 \\
7 \cdot 02 \\
7 \cdot 56\end{array}$ & $\begin{array}{c}68 \cdot 89^{* * *} \\
8 \cdot 14 \\
23 \cdot 70^{*} \\
3 \cdot 3^{8} \\
17 \cdot 05^{*}\end{array}$ \\
\hline Total & & 24 & & & & & & & & & \\
\hline $\begin{array}{l}\mathbf{R} \\
\mathbf{R} t\end{array}$ & - & $\begin{array}{r}1 \\
22\end{array}$ & $\begin{array}{l}8 \cdot 83 \\
6 \cdot 75\end{array}$ & $\begin{array}{l}3 \cdot 71 \\
6 \cdot 46\end{array}$ & $\begin{array}{l}0 \cdot 62 \\
5 \cdot 28\end{array}$ & $\begin{array}{l}0.78 \\
3.47\end{array}$ & $\begin{array}{l}0.86 \\
7.09\end{array}$ & $\begin{array}{l}0.11 \\
7.51\end{array}$ & $\begin{array}{l}3 \cdot 83 \\
5 \cdot 13\end{array}$ & $\begin{array}{l}2 \cdot 80 \\
7 \cdot 20\end{array}$ & $\begin{array}{l}1 \cdot 53 \\
5 \cdot 09\end{array}$ \\
\hline $\begin{array}{c}\text { Grand } \\
\text { total }\end{array}$ & & 47 & & & & & & & & & \\
\hline
\end{tabular}

TABLE 3

The analyses of the results from the experiment carried out at $25^{\circ} \mathrm{C}$.

Other details as in previous tables

\begin{tabular}{|c|c|c|c|c|c|c|c|c|c|c|c|}
\hline \multicolumn{2}{|c|}{ Items } & d.f. & I & 2 & 3 & 4 & 5 & $5 \mathrm{~A}$ & ${ }_{5} B$ & 6 & Totals \\
\hline $\begin{array}{ll}a & \\
b & : \\
b_{1} & : \\
c & : \\
d & :\end{array}$ & . & $\begin{array}{r}4 \\
10 \\
1 \quad \\
4 \\
6\end{array}$ & $\begin{array}{c}29 \cdot 80^{* *} \\
8 \cdot 77 \\
0 \cdot 20 \\
8 \cdot 36 \\
3 \cdot 32\end{array}$ & $\begin{array}{c}34.22^{*} \\
13.53 \\
40.10 \\
6.32 \\
4.85\end{array}$ & $\begin{array}{c}9 \cdot 64 \\
10 \cdot 4^{8} \\
69 \cdot 96^{* *} \\
6 \cdot 64 \\
6 \cdot 46\end{array}$ & $\begin{array}{l}8 \cdot 44 \\
7 \cdot 05 \\
0 \\
5 \cdot 55 \\
7 \cdot 97\end{array}$ & $\begin{array}{r}11.03 \\
5.07 \\
0.66 \\
5.53 \\
17.60\end{array}$ & $\begin{array}{r}3.38 \\
1.87 \\
0.26 \\
13.16 \\
2.16\end{array}$ & $\begin{array}{c}9 \cdot 85 \\
10 \cdot 30 \\
14 \cdot 77 \\
10 \cdot 26 \\
22 \cdot 74^{* *}\end{array}$ & $\begin{array}{r}11 \cdot 32 \\
6 \cdot 43 \\
3.52 \\
1.64 \\
8.37\end{array}$ & $\begin{array}{r}20 \cdot 59 \\
9 \cdot 39 \\
37 \cdot 30 \\
5 \cdot 49 \\
6 \cdot 33\end{array}$ \\
\hline Total & . & 24 & & & & & & & & & \\
\hline $\begin{array}{l}\mathrm{R} \\
\mathrm{R} t\end{array}$ & $\cdot$ & $\begin{array}{r}1 \\
23\end{array}$ & $\begin{array}{l}2 \cdot 97 \\
4.89\end{array}$ & $\begin{array}{l}11.07 \\
10.30\end{array}$ & $\begin{array}{c}47^{\circ} 45^{*} \\
7.50\end{array}$ & $\begin{array}{l}0 \cdot 14 \\
5 \cdot 78\end{array}$ & $\begin{array}{l}0.21 \\
9 \cdot 72\end{array}$ & $\begin{array}{c}29.3 \mathrm{~J}^{*} \\
6.06\end{array}$ & $\begin{array}{l}14 \cdot 30 \\
14 \cdot 46 \text { ** }\end{array}$ & $\begin{array}{l}20 \cdot 11 \\
13 \cdot 13\end{array}$ & $\begin{array}{r}6.77 \\
13.41\end{array}$ \\
\hline Grand & tal & $4^{8}$ & & & & & & & & & \\
\hline
\end{tabular}

no evidence of any additive genetic variation with respect to recombination frequency at $25^{\circ} \mathrm{C}$. In short, the widespread variation of this type shown in the combined analysis (table IA) stems almost entirely from the results gained at $18^{\circ} \mathrm{C}$. It is hardly surprising therefore that, as already noted, there is no evidence of genotype-environment interaction (with the exception of region 6). There is so little interpretable 
variation between genotypes at $25^{\circ} \mathrm{C}$. that the analysis of totals shows no significant mean squares. At $18^{\circ} \mathrm{C}$. on the other hand, there are marked differences between arrays, some dominance and, apparently, an overall difference between reciprocal FI's of the type ascribable to maternal effects. Since the $b_{1}$ item is significant in none of the segment analyses, at first sight this suggests a weak overall superiority of the FI's when compared to their parents. In fact, as fig. 5 shows dominance is localised distally as it is at $25^{\circ} \mathrm{C}$. Lastly, with regard to reciprocal differences it is of some interest that region ${ }_{5} \mathrm{~B}$ shows significant evidence of this in both environments although not from the analysis on data gained by pooling over environments (table IA). In the latter, however, it will be recalled that the $\mathrm{E} d$ item was highly significant. Thus this region provides stronger evidence for the effect of environmental variation on the expression of maternal effects than was forthcoming from region 3 .

\section{DISCUSSION}

The results of the first and second experiments agree in showing that crossing over is subject to genetic control and in this respect extend the evidence gained from a number of sources ( $c f$. Rees, $196 \mathrm{I}$ ). The design of the present experiment, however, allows a more detailed evaluation of this control, both with regard to its nature and its distribution along the length of the chromosome concerned.

As regards the nature of the genetic control, three main points emerge. Firstly, the genic content of chromosomes other than that whose crossing over is observed is of importance to crossing over in that chromosome. Part, at least, of the genetic control of crossing over within any chromosome is thus exercised at an inter-chromosomal level. In this connection the results of Levine and Levine (1954, 55) from Drosophila pseudoobscura are similar to those presented here. They were able to show that the effect of various third chromosome rearrangements on crossing over in the X-chromosome of that species could not be solely ascribed to the "mechanical " properties of these rearrangements, their genic content being of importance too. There are, no doubt, some genes concerned with the control of crossing over within the chromosome on which they are located as Parson's (1957) and Law's (196I) results would indicate. The importance of intrachromosomal control relative to that exercised at the inter-chromosomal level appears, however, not to be known.

Secondly, the evidence of inter-chromosomal control of crossing over alone indicates that the genetic system concerned is likely to be polygenic in kind as the results of Rees (1955), Rees and Thompson (1956) and Gowen (1919) indicate. More direct evidence from the present results, while less compelling than these, provide no hint of simple differences between lines. There is rather, a range of recombination frequencies of which Edinburgh and Florida appear to be the extremes. It would indeed be surprising if the genetic control of crossing over was not of a polygenic nature, for simple differences of 
the type determined by major genes could hardly be expected to provide an adequate basis for the fine adjustment by natural selection, which the conflicting requirements of fitness and flexibility demand (Darlington, I958).

The third point of interest here concerns the components of the genetic system concerned with the control of crossing over. The present results show that the additive component accounts for nearly all the measurable genetic variance, there being rather little dominance and no detectable genic interaction. In so far that Detlefsen and Roberts (I92I) were able to select successfully for low recombination frequency in Drosophila melanogaster, the genetic variance of their base population must have contained a substantial additive component. Rees and Thompson (loc. cit.) on the other hand were able to show that in rye all three of these components were of importance in the genetic system of control of crossing-over. The results of Levine and Levine (loc. cit.) also indicate the importance of these components. A reanalysis of their data shows that of the two regions of the $\mathrm{X}$-chromosome of Drosophila pseudoobscura they examined, the most distal $(y-s n)$ displays clear evidence of both additive and dominance variation, with the direction of dominance being towards high frequency of recombination. Most of the dominance variation lies apparently between the standard third chromosome (ST) on the one hand and the two inversion chromosomes (AR and $\mathrm{CH}$ ) on the other. There is considerably less variation between the strains of these chromosomes. The other region of the X-chromosome they examined $(s n-v)$ provides less evidence of heritable variation, although unlike the adjacent segment there is some indication of genic interaction (which like dominance is also in the direction of high frequency of recombination). A more detailed analysis of their results would be of considerable interest but is not possible on their published information.

Evidently, though there is a paucity of information concerning the components of variation of the genetic system here, all three major components can be of importance. We need, therefore, to ask why additive variation alone has been detected in the present experiment.

Now while Drosophila melanogaster in the wild is no doubt a characteristically outbreeding species, the lines used here all have long histories of laboratory culture. Under these conditions there would appear to be little requirement for free heritable variation so that selection might be expected to be basically stabilising in its action (Mather, I953). In fact, as has been mentioned, less genetic variation was detected at $25^{\circ} \mathrm{C}$. than at $18^{\circ} \mathrm{C}$. This is to be expected, since if selection has been effective in the way suggested, then it will have been at $25^{\circ} \mathrm{C}$., the standard laboratory environment, while the $18^{\circ} \mathrm{C}$. environment is novel in this respect. On this view, the homogeneity of the laboratory environment at $25^{\circ} \mathrm{C}$. has caused all lines to have been adjusted by selection to a level of crossing over presumably optimal for these conditions. Furthermore, where selection is, in the 
main, stabilising in its action, dominance and interaction effects are likely to be balanced in their action (Mather, 196r). This interpretation is particularly relevant here since we are concerned with genetic differences between whole chromosomes, no opportunity for recombination between them having been allowed. It is possible that if the experiment had been carried on to the $F_{2}$ generation some of this potential variation would have been released by recombination and hence capable of detection.

On the distribution of the genetic control of crossing over both experiments agree in showing that this is effective along the entire length of the X-chromosome. As we have seen, this is largely additive in kind, a little dominance being detected in the distal regions 2 and 3 . This situation could be due to either or both of two different aspects of the control of crossing over. That most regions display the importance of the genetic component of control of crossing over may be but a simple consequence of the mechanism of crossing over. On Mather's (1938) view, the formation of chiasmata is a serial process, the first chiasma being formed near the centromere while those subsequent to this are then formed in more distal regions. In this way the distributions of distal chiasmata are not independent of those located nearer the centromere. Thus if the latter are subject to genetic control, the others will appear to be also.

On the other hand, it is conceivable that genetic control of crossing over is to some extent at least independent in adjacent segments of the chromosome. In this case it should be possible for natural selection to adjust the frequency of recombination in either segment to whatever constitutes an optimal level.

We are not able to explore these alternatives with the present results, but those of Detlefsen and Roberts (loc. cit.) and Detlefsen and Clemente (1923) appear to support the first of these. These authors were able to select for a low frequency of recombination in the white-eye $(w)$, miniature-wing $(m)$ region of the X-chromosome of Drosophila melanogaster. Examination of the effects of selection in this region on the frequency of recombination in the adjacent and more proximal region $(m-f)$ showed that here too the amount of crossing over had been markedly reduced. On the second view there is no a priori reason why this result should have been obtained. But on the first view the results obtained in the $w-m$ segment must have been obtained at one remove; i.e. the primary effect of selection must have been on the frequency of recombination in the $m-f$ region, the change observed in that of the $w-m$ region being a consequence of change secured in the proximal region. Further experiments along the lines of that carried out by these authors could clearly be of value here and it is surprising that this appears to be the only one reported.

While the results of the present experiments have in the main accorded well with the results of previous authors, the effect of change of environment on the frequency of recombination has not. It has been 
a general observation that only proximal regions of the chromosome are sensitive to changes of the environment whether these be concerned with temperature, ionising radiation or indeed, maternal effects ( $c f$. Mather, I938). Further, Mather's (1939) results suggested that this property is attributable to the heterochromatin whose abundance is a characteristic of these regions of Drosophila chromosomes. It is difficult to reconcile the present results with this view for, as we have seen crossing over in most regions of the $\mathrm{X}$-chromosome responds to a change of temperature, only the most proximal and the most distal regions failing to respond in this way. Only if it could be argued that the X-chromosomes used here had highly unusual distributions of heterochromatin would it be possible to interpret the present results in this way-and there is no evidence to support this argument. It thus seems more likely that sensitivity to the environment is mediated by agencies other than that of heterochromatin, whatever these may be.

It is, however, worth pointing out that in earlier experiments inbred lines were rarely used, though various attempts to secure homogeneity of the material were made from time to time (e.g. by using sibs). In short, are the present results to be explained in terms of the relatively greater instability of the inbred parents, when these are compared with their F I progeny, with regard to changes of environment? The analyses presented so far do not allow a partitioning of the appropriate items needed to answer this question. However, a preliminary answer might be gained by enquiring whether the FI's are on average less sensitive to changes of the environment than their inbred parents. This comparison is presented graphically in fig. 5 and it is at once obvious that both parents and FI's respond similarly to the same change of environment.

It is just possible, however, that while on average the FI's are no less sensitive to changes of the environment than their inbred parents, detailed comparisons between particular parents and their F I crosses might show the latter to be less sensitive. The analysis required for this purpose is shown in table 4 . In this, the 24 degrees of freedom available from the results of a $5 \times 5$ diallel have been partitioned into an item estimating variation between parent lines $(\mathrm{P})$ with 4 degrees of freedom and another estimating variation between FI's with I9 degrees of freedom $(F)$. The remaining degree of freedom is concerned of course with the comparison between the parents and their FI's and is the $b_{1}$ item of previous analyses. Item $\mathbf{F}$ may be partitioned into a sum of squares estimating variation between reciprocal sums $\left(\mathbf{F}_{S}\right)$ with, 9 degrees of freedom and another concerned with reciprocal differences $\left(F_{D}\right)$ with Io degrees of freedom. Again the $F_{D}$ item appears in previous analyses as the sum of the $c$ and $d$ items. It follows, therefore, that the $\mathrm{P}$ and $\mathrm{F}_{\boldsymbol{S}}$ sums of squares are obtained by a new partitioning of the $a, b_{2}$ and $b_{3}$ items of Hayman's analysis. In biometrical terms, item $\mathrm{P}$ is an estimate of $\mathrm{D}$, the additive component of genetic variation (Mather, 1949), together with the usual environmental component, E. 
The $\mathrm{F}_{S}$ item is a sum of squares estimating $\mathrm{D}, \mathrm{H}$ and $\mathrm{E}$, the remaining items carrying the same interpretation as in Hayman's analysis. In other respects the analysis is identical with those presented earlier.

The items most pertinent to our enquiry about the relative sensitivities of the parents and their $\mathrm{F}_{\mathrm{I}}$ 's over environments are $\mathrm{EP}$ and $\mathrm{EF}_{S}$. From these it is clear that there is very little difference concerning the environmental sensitivities of crossing over between parents and their

TABLE 4

Analysis of variance of transformed recombination frequencies pooled over sexes (variation between cultures). The M.S.'s in the table are gained by a new partitioning of the items in the last column of table IA (see text)

\begin{tabular}{|c|c|c|}
\hline Items & d.f. & M.S. \\
\hline $\begin{array}{l}\mathbf{P} \\
\mathbf{F} \\
\mathbf{F}_{S} \\
\mathbf{F}_{D} \\
\mathbf{P}_{-\mathbf{F}}\end{array}$ & $\begin{array}{rr} & 4 \\
& 19 \\
9 & \\
10 & \\
& 1\end{array}$ & $\begin{array}{c}26 \cdot 19^{*} \\
14.97 \\
22 \cdot 88^{*} \\
7 \cdot 86 \\
60 \cdot 23^{*}\end{array}$ \\
\hline Total . & 24 & \\
\hline $\begin{array}{l}\mathbf{R} \\
\mathbf{R P} \\
\mathbf{R F}_{S}:\end{array}$ & $\begin{array}{l}\text { I } \\
4\end{array}$ & $\begin{array}{r}0.93 \\
18.03 \\
7.92\end{array}$ \\
\hline $\mathrm{RT}$. & 24 & \\
\hline$E$ & I & $583 \cdot 77^{* * *}$ \\
\hline $\begin{array}{l}\mathrm{EP} \\
\mathrm{EF}_{S}\end{array}$ & 4 & $\begin{array}{l}11 \cdot 05 \\
13 \cdot 03\end{array}$ \\
\hline $\mathrm{E} t$ & 24 & \\
\hline $\mathrm{RE}$. & I & $7 \cdot 37$ \\
\hline $\mathrm{RE} t$ & 21 & $9 \cdot 22$ \\
\hline Grand total & 96 & \\
\hline
\end{tabular}

FI's-the mean square for the latter actually being a little larger than that for their parents. There appears, therefore, to be no evidence to support the view that the response to change of environment of crossing over in this experiment depends on differences between parents and their FI's in this respect.

As we have seen, maternal effects are trivial and elusive here in comparison with those found in the first experiment. Now in the latter, temperature treatment was restricted to the larval and pupal stages of the triply heterozygous females. In the second experiment, the period of treatment was extended to include the first two days at $25^{\circ} \mathrm{C}$. and the first four days of adult life at $18^{\circ} \mathrm{C}$. If, as Bodenstein (1950) states, meiosis in female Drosophila individuals occurs on the 
emergence of the imago from the pupa, (or at least shortly after), then in the second experiment the period of treatment covered both premeiotic and the meiotic divisions while the first experiment was concerned with the pre-meiotic stages of oogenesis only. It is possible, although not perhaps very likely, that the environmental variance induced by the treatment of the meiotic divisions has swamped that induced in preceding stages of oogenesis. It is not of course possible to proceed further here but treatment fractionation experiments along these lines would clearly be of interest.

\section{SUMMARY}

I. Five inbred lines of Drosophila melanogaster were crossed in all combinations and the frequency of recombination between eight marker genes carried on a common pair of X-chromosomes was scored among the backcross progenies of the parent lines and their twenty FI's. The experiment was replicated and carried out in two environments provided by incubators set at $18^{\circ} \mathrm{C}$. and $25^{\circ} \mathrm{C}$.

2. As regards the nature of the genetic control of crossing over, three points emerge from the results:-

(i) part of the control is exercised at the inter-chromosomal level;

(ii) control is polygenic; and

(iii) of the three main components of genetic variation, the additive component is of greatest importance, there being only slight dominance and no genic interaction.

3. The results also indicate that crossing over in most parts of the $\mathrm{X}$-chromosome is under genetic control.

4. The effect of change of environment on the frequency of recombination is not confined to those regions of the chromosome near the centromere, most regions displaying this effect but not that adjacent to the centromere. Environmental sensitivity therefore appears to be unrelated to the distribution of heterochromatin; nor is it related to the differential sensitivities of the inbred parents and their FI's.

5. There is very little evidence of maternal control of crossing over.

Acknowledgments. - I am indebted to Dr. J. L. Jinks for his advice and encouragement throughout the course of this work and to Professor K. Mather for his advice on the preparation of this account for publication.

\section{REFERENCES}

ALLARD, R. W., AND ALDER, H. L. I960. The effect of incomplete penetrance on the estimation of recombination values. Heredity, $15,263-82$.

bodenstein, D. 1960. In Biology of Drosophila; ed. Demerec, John Wiley, New York.

BREESE, E. L., AND MATHER, K. 1957. The organisation of polygenic activity within a chromosome in Drosophila. I. Hair characters. Heredity, II, 373-95.

BRIDGES, C. B., AND BREHME, x. s. 1944. The mutants of Drosophila melanogaster Carnegie Institution of Washington Publication $55^{2}$. 
DARLINGton, C. D. 1958. Evolution of genetic systems. Oliver and Boyd, Edinburgh. DETLEFSEN, J. A., AND CLEMENTE, L. S. 1923. Genetic variation in linkage values. Proc. Nat. Acad. Sci. (Wash.), 9, 149-56.

DETLEFSEN, J. A., AND ROBERTs, E. I 921 . Studies on crossing over. I. The effect of selection on crossover values. F. Exp. Zoology, 32, 333-54.

rOWEN, J. W. 1919. A biometrical study of crossing over. On the mechanism of crossing over in the third chromosome of Drosophila melanogaster. Genetics, 4, 205-50.

haYMAN, B. I. 1954. The analysis of variance of diallel tables. Biometrics, 1o, 235-44.

JINks, J. L. 1954. The analysis of continuous variation in a diallel of Nicotiana rustica varieties. Genetics, 39, 767-88.

LAW, C. N. I96I. Recombination in the X-chromosome of Drosophila melanogaster. Nature, 191, $1180-81$.

LAWRENCE, M. J. 1958. Genotypic control of crossing over on the first chromosome of Drosophila melanogaster. Nature, 182, 889-9o.

LEVINE, R. P., AND LEVINE, E. P. 1954. The genotypic control of crossing over in Drosophila pseudoobscura. Genetics, 39, 677-91.

LEVINE, R. P., AND LEVINE, E. P. 1955. Variable crossing over arising in different strains of Drosophila pseudoobscura. Genetics, 40, 339-405.

mather, K. 1939. Crossing over. Biol. Rev., 13, 258-92.

MATHER, K. 1939. Crossing over and heterochromatin in the X-chromosome of Drosophila melanogaster. Genetics, 24, 413-35.

MAtHer, K. 1949. Biometrical Genetics. Methuen, London.

MATHER, K. I953. The genetical structure of populations. Symp. Soc. Exp. Biol., 7, 66-95.

MATHER, K. 1960. Evolution in polygenic systems. Evolutzione e genetica, 47, 131-52. PARsONS, P. A. 1957. An effect of gene arrangement on the recombination fraction in Drosophila melanogaster. Heredity, 11,11 7-27.

PARsons, P. A. 1957. Partial manifestation of a gene in complete three and higher point backcross data. Heredity, 11, 21 7-22.

REES, M. 1955. Genotypic control of chromosome behaviour in rye. I. Inbred lines. Heredity, 9, 93-1 16.

REES, H., AND THOMPSON, J. B. 1956. Genotypic control of chromosome behaviour in rye. III. Chiasma frequency in homozygotes and heterozygotes. Heredity, 10, 402-24.

REEs, M. 196r. Genotypic control of chromosome form and behaviour. Bot. Rev., 27, 288-318.

WALLACE, M. E. 1957. A balanced three-point experiment for linkage group V of the house mouse. Heredity, II, 223-58.

WIGAN, L. G. 1949. The distribution of polygenic activity on the X-chromosome of Drosophila melanogaster. Heredity, 3, 53-66. 